
Not for reproduction, distribution or commercial use.

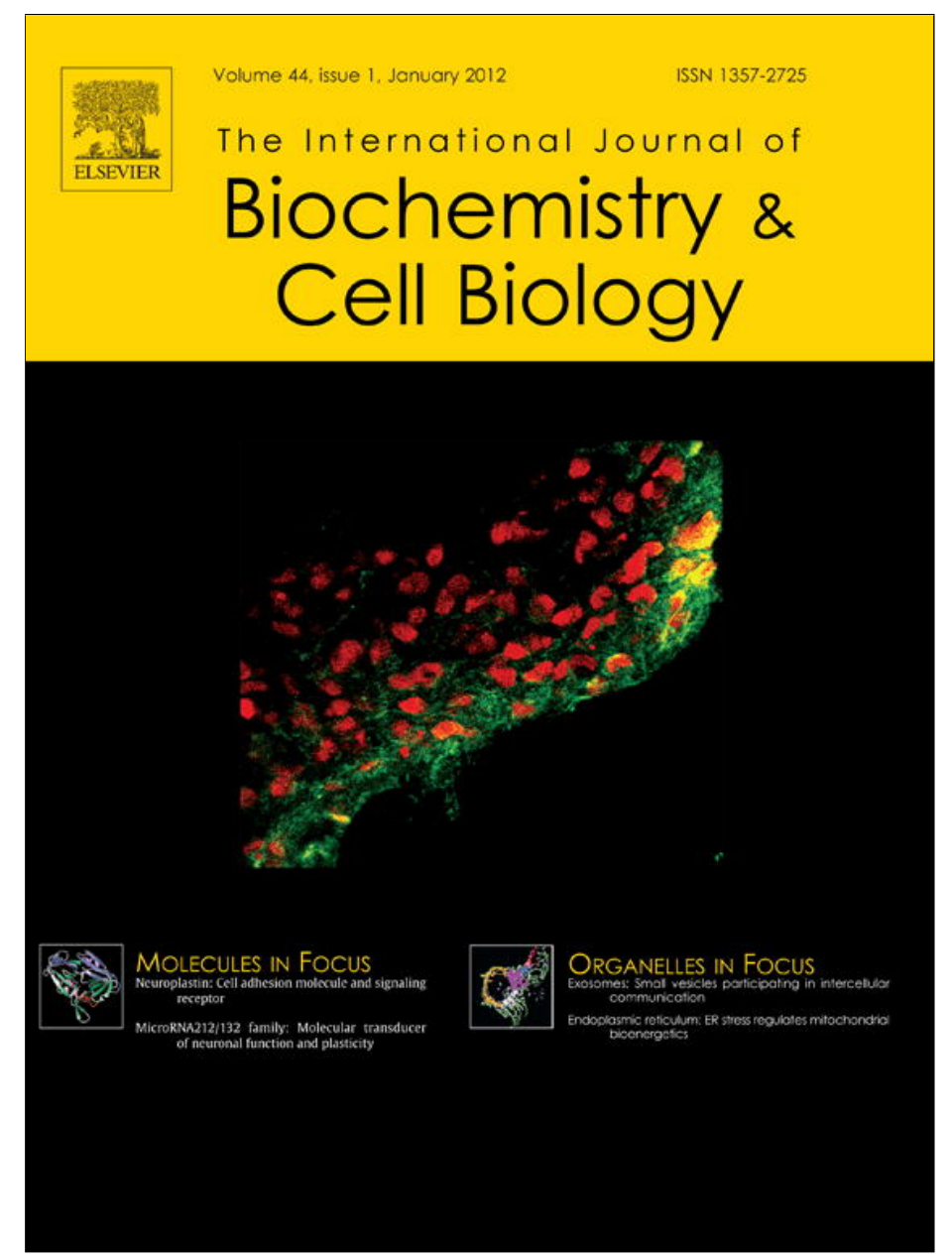

(This is a sample cover image for this issue. The actual cover is not yet available at this time.)

This article appeared in a journal published by Elsevier. The attached copy is furnished to the author for internal non-commercial research and education use, including for instruction at the authors institution and sharing with colleagues.

Other uses, including reproduction and distribution, or selling or licensing copies, or posting to personal, institutional or third party websites are prohibited.

In most cases authors are permitted to post their version of the article (e.g. in Word or Tex form) to their personal website or institutional repository. Authors requiring further information regarding Elsevier's archiving and manuscript policies are encouraged to visit:

http://www.elsevier.com/copyright 


\title{
An active mitochondrial biogenesis occurs during dendritic cell differentiation
}

\author{
Patrizia Zaccagnino ${ }^{a}$, Maddalena Saltarella $^{a}$, Stefania Maiorano $^{a}$, Antonio Gaballo $^{b}$, Giuseppe Santoro $^{c}$, \\ Beatrice Nico a ${ }^{a}$ Michele Lorusso ${ }^{\mathrm{a}, *}$, Annalisa Del Prete ${ }^{\mathrm{a}, \mathrm{d}, * *}$ \\ ${ }^{a}$ Department of Basic Medical Sciences, University of Bari, Piazza G. Cesare 11, 70124 Bari, Italy \\ ${ }^{\mathrm{b}}$ Institute of Biomembrane and Bioenergetics, CNR, Piazza G. Cesare, 70124 Bari, Italy

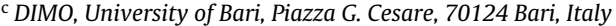 \\ ${ }^{\mathrm{d}}$ Humanitas Clinical and Research Center, via Manzoni 56, 20089 Rozzano (MI), Italy
}

\section{A R T I C L E I N F O}

\section{Article history:}

Received 6 March 2012

Received in revised form 3 July 2012

Accepted 24 July 2012

Available online $\mathrm{xxx}$

\section{Keywords:}

Dendritic cell differentiation

Mitochondrial respiratory complexes

Mitochondrial biogenesis

Anti-oxidant enzymes

\begin{abstract}
A B S T R A C $T$
Dendritic cells (DC) are sentinels of the immune system deriving from circulating monocyte precursors recruited to sites of inflammation. In a previous report (Del Prete et al., 2008) we showed that, after differentiation, DC exhibited increased number of condensed mitochondria and dynamic changes in their energy metabolism. A study is presented here showing that the DC differentiation process is characterized by increased expression level and activity of mitochondrial respiratory complexes, as well as by an increased mitochondrial DNA (mtDNA) copy number. Moreover, DC are equipped with more efficient antioxidant protection systems, over expressed most likely to detoxify increased ROS production, as a consequence of the much higher mitochondrial activity. Kinetic analysis of the three main mitochondrial biogenesis-associated genes revealed that the peak in PPAR $\gamma$ coactivator-1alpha (PGC-1 $\alpha)$ gene expression was suddenly reached few hours after the onset of the differentiation. While PGC-1 $\alpha$ expression rapidly declines, the mitochondrial transcription factor A (TFAM) and nuclear respiratory factor-1 (NRF-1) expression gradually increased. These findings demonstrate that an active mitochondrial biogenesis occurs during DC differentiation and further suggest that an early input by the master regulator of mitochondrial biogenesis PGC $-1 \alpha$ is needed to trigger the subsequent activation of the downstream transcription factors, NRF-1 and TFAM in this process.
\end{abstract}

(C) 2012 Elsevier Ltd. All rights reserved.

\section{Introduction}

Dendritic cells are professional antigen presenting cells that play a critical role in the initiation and regulation of adaptive immune responses (Steinman, 2003). DC derive from bone marrow progenitors or from monocytes and then either stay in the blood stream or migrate into peripheral tissues, where they show an immature phenotype. Immature DC, such as Langerhans cells in the skin, act as sentinels, continuously taking up antigens and undergoing activation (Banchereau et al., 2000). During their maturation, DC migrate to secondary lymphoid organs, where they become specialized immune-stimulatory cells able to activate naive T cells (Sozzani, 2005).

In a previous report, we showed that the differentiation process of peripheral blood monocytes to DC requires growth factor

\footnotetext{
* Corresponding author. Tel.: +39 0805448522; fax: +39 0805448538

** Corresponding author at: Department of Basic Medical Sciences, University of Bari, Piazza G. Cesare 11, 70124 Bari, Italy. Tel.: +39 0805448522; fax: +390805448538

E-mail addresses: m.lorusso@biochem.uniba.it (M. Lorusso), delpreteanna@hotmail.com (A. Del Prete).
}

dependent production of intracellular Reactive Oxygen Species (ROS) (Del Prete et al., 2008; see also Sattler et al., 1999; Sheng et al., 2010). As revealed by ultrastructural analysis, DC exhibited an increased number of condensed mitochondria compared to monocyte precursors. A higher endogenous respiratory activity together with an increase in ATP content and mitochondrial enzyme citrate synthase activity was also found. Moreover, the presence of mitochondrial Complex I inhibitor rotenone into the culture medium caused inhibition of the DC differentiation process, through inhibition of mitochondrial ROS production (Del Prete et al., 2008).

Mitochondria are best known for their role in ATP production through oxidative phosphorylation, calcium homeostasis, apoptosis and cell signalling (Kroemer and Reed, 2000; Chan, 2006). Furthermore, mitochondria are the major source of endogenous ROS that play a key role in cell physiology including cell differentiation and proliferation (Kiefel et al., 2006). Mitochondria possess their own genomic apparatus, mtDNA, a $16.5 \mathrm{~kb}$ closed-circular double stranded DNA, which codes for some of their proteins. Consequently, mitochondrial biogenesis is highly orchestrated by the transcriptional regulatory circuits between this mtDNA and nuclear genes encoding mitochondrial proteins (Garesse and Vallejo, 2001; Kelly and Scarpulla, 2004). Transcription of mtDNA requires, in addition to the mitochondrial specific DNA polymerase gamma 
(POLG), a small number of nucleus-encoded proteins comprising the mitochondrial transcription factor A (TFAM) (Clayton, 1998; Scarpulla, 2002). Additional regulating elements include the transcription factor NRF- 1 and members of the PGC- 1 family (Scarpulla, 2008). In particular, PGC- $1 \alpha$ appears as a master regulator of mitochondria biogenesis due to its ability to activate TFAM through direct interaction with NRF-1 (Goffart and Wiesner, 2003; Scarpulla, 2011).

In the present study the process of mitochondrial biogenesis accompanying the differentiation of monocyte precursors in DC was examined. In particular, we focused on the structural and functional changes in mitochondrial respiratory complexes and the expression and activity of anti-oxidant enzymes occurring during this process. Moreover, a direct comparison of the expression level of mitochondrial proteins was performed by using a new technical approach which allows pure mitochondrial fraction to be isolated from both monocytes and DC. Finally, the mtDNA content and the gene expression changes occurring during the monocyte to DC transition were studied, together with the kinetics of the transcription of mitochondria-related genes over the 6 -days culture period.

\section{Materials and methods}

\subsection{Dendritic cell generation and characterization}

Peripheral blood mononuclear cells (PBMC) were isolated by the standard Ficoll-Paque (Amersham, UK) gradient centrifugation from buffy coats (obtained through the courtesy of the local Blood Bank, Polyclinic Hospital, Bari, Italy). Monocytes were purified by immunomagnetic separation using anti-CD14 conjugated magnetic microbeads (Miltenyi Biotec, Bergisch Gladbach, Germany) and cultured for 6 days at $1 \times 10^{6} / \mathrm{ml}$ in RPMI 1640 medium (Biochrom AG, Leonorenstr, Berlin) supplemented with heat-inactivated 10\% FBS (EuroClone, Milan, Italy), GM-CSF (50 ng/ml) and IL-4 $(20 \mathrm{ng} / \mathrm{ml})$ (R\&D Systems, Minneapolis, MN). Surface phenotype analysis was performed using human Mo-DC differentiation inspector, containing monoclonal antibodies recognizing CD14, CD83 and CD209 and corresponding isotype controls (Miltenyi Biotec).

\subsection{Mitochondria isolation with superparamagnetic microbeads}

Mitochondria from monocytes and DC were purified by using the superparamagnetic anti-translocase of outer mitochondrial membrane 22 (-TOM22) microbeads (Miltenyi Biotec). Briefly, monocytes and DC were washed twice with phosphate-buffered saline (PBS), and lysed by shearing through a needle (29G) approximately 40 times. After cell disruption, an aliquot of the lysate was examined for trypan blue exclusion to ensure that $80 \%$ of the cells were lysed. For magnetic labelling, cell lysate was incubated with anti-TOM22 microbeads for $60 \mathrm{~min}$ at $4{ }^{\circ} \mathrm{C}$, with gentle shaking. Subsequently, cell suspension was loaded onto a pre-equilibrated MACS column, and finally, retained mitochondria were eluted. Following centrifugation at $16,000 \times g$ for $1 \mathrm{~min}$, the mitochondrial pellet was finally resuspended in the appropriate storage buffer.

\subsection{Measurement of respiratory complex activities}

Monocytes and DC were suspended in a medium contain-

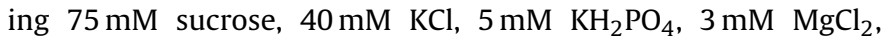
$0.5 \mathrm{mM}$ EDTA and $30 \mathrm{mM}$ Tris- $\mathrm{HCl}$ (pH 7.4) (Buffer A) and supplemented with $30 \mu \mathrm{g} / 10^{6}$ cells digitonin as previously described (Sgobbo et al., 2007). After 2 min, cell suspension was centrifuged and the pellet was resuspended in hypotonic medium containing $25 \mathrm{mM} \mathrm{KH}_{2} \mathrm{PO}_{4}(\mathrm{pH} 7.2$ ), $5 \mathrm{mM} \mathrm{MgCl} 2$ and the anti-protease cocktail tablet (Roche, Basel, CH) (Buffer B). After freezing and thawing three times, the protein concentration was determined.
Enzyme activities were measured using a Beckmann DU7400 spectrophotometer. Complex I activity was assayed by following the rotenone-sensitive initial rate of NADH oxidation at 340-425 nm $\left(\Delta \varepsilon=6.81 \mathrm{mM}^{-1} \mathrm{~cm}^{-1}\right) .200 \mu \mathrm{g} / \mathrm{ml}$ protein were added to Buffer B containing $2.5 \mathrm{mg} / \mathrm{ml}$ of BSA, $2.2 \mathrm{mM} \mathrm{KCN}, 3.6 \mu \mathrm{g} / \mathrm{ml}$ antimycin A and $71 \mu \mathrm{M}$ decylubiquinone. The reaction was started by the addition of $68 \mu \mathrm{M}$ NADH followed by $1.8 \mu \mathrm{g} / \mathrm{ml}$ rotenone. Complex IV activity was measured following the oxidation of ferrocytochrome c at $550-540 \mathrm{~nm}\left(\Delta \varepsilon=19.1 \mathrm{mM}^{-1} \mathrm{~cm}^{-1}\right) .50 \mu \mathrm{g} / \mathrm{ml}$ of protein were added to Buffer B supplemented with $3.3 \mu \mathrm{g} / \mathrm{ml}$ antimycin A and $500 \mu \mathrm{M}$ dodecyl maltoside. The reaction was started by the addition of $9 \mu \mathrm{M}$ reduced cytochrome $\mathrm{c}$, in the absence and in the presence of $1.6 \mathrm{mM} \mathrm{KCN}$.

\subsection{Measurement of intracellular ATP}

ATP concentration was determined using a bioluminescence somatic cell assay kit, following the manufacturer's instructions. $2 \times 10^{4}$ cells were used for each sample. Chemiluminescence signals were acquired with a Victor X3 Multilabel Plate Readers (PerkinElmer, San Josè, CA, USA).

\subsection{Measurement of lactate production}

Analysis of lactate released into the culture medium was carried out spectrophotometrically by following $\mathrm{NAD}^{+}$reduction at $340 \mathrm{~nm}$, as previously described (Del Prete et al., 2008).

\subsection{Anti-oxidant enzyme activities}

Superoxide dismutase (SOD) activity was measured using the SOD assay kit (Cayman Chemical, Ann Arbor, MI), according to the manufacturer's instructions. Briefly, monocytes and DC were centrifuged at $16,000 \times \mathrm{g}$ for $1 \mathrm{~min}$ and the cell pellet was homogenized using a 29G needle and centrifuged at $1500 \times \mathrm{g}$ for $5 \mathrm{~min}$ at $4{ }^{\circ} \mathrm{C}$. Finally, the supernatant was used for analysis of SOD activity. The addition of $4 \mathrm{mM} \mathrm{KCN}$ to the assay was used to inhibit both CuZn- and extracellular-SOD, thus allowing the detection of the activity of MnSOD only. One unit of SOD was defined as the amount of enzyme catalysing 50\% dismutation of the superoxide radical. Absorbance change was recorded at $450 \mathrm{~nm}$ using a Victor X3 Multilabel Plate Readers. Catalase activity was assayed by monitoring spectrophotometrically the rate of decomposition of $\mathrm{H}_{2} \mathrm{O}_{2}$ at $240 \mathrm{~nm}$ as previously described (Pacelli et al., 2011).

\subsection{Western blotting analysis}

Monocytes and DC or isolated mitochondria were separated on $12 \%$ Tris-Tricine SDS-PAGE and blotted onto nitrocellulose membrane. Western blot (WB) analysis was carried out using the following primary antibodies: Fe-S protein 3 (NDUFS3) of Complex I (MitoSciences Inc.; Eugene, OR, USA), Core I subunit of Complex III, COX IV of Complex IV and porin (VDAC) (Invitrogen, Carlsbad, CA, USA), TOM22 and TFAM (GeneTex, Inc., Irvine, CA, USA), MnSOD and CuZnSOD (Millipore, Billerica, MA, USA), Catalase (Calbiochem, San Diego, CA, USA), and GAPDH (AbD Serotec, Dusseldorf, Germany), NRF-1 (Abcam, Cambridge, UK) according to the manufacturers' suggested concentrations. Proteins were detected by chemiluminescent LiteAblot reagent (EuroClone) and the relative optical band densities were quantified by densitometric analysis using Quantity One-4.4.1 imaging software (Bio-Rad Laboratories).

\section{8. mtDNA quantification}

For determination of the mtDNA copy number, total DNA was isolated from $5 \times 10^{6}$ monocytes and DC using EuroGOLD Tissue 
DNA Mini Kit (Euroclone). Content of mtDNA per cell was measured by using human mitochondrial DNA qPCR Detection kit (Gene More s.r.l., Mo, Italy).

\subsection{Real-time quantitative reverse transcription-PCR}

Transcript levels were quantified by real-time reverse transcription-PCR by extracting total RNA using Trizol (Invitrogen) from monocytes and DC. RNA was then further purified and DNase treated (Roche). First-strand cDNA was synthesized from $1 \mu \mathrm{g}$ of total RNA using iScript ${ }^{\mathrm{TM}}$ CDNA Synthesis Kit (Bio-Rad) and Oligo dT-primers, according to the manufacturer's instructions. mRNA expression for genes of interest was performed by RT real-time PCR with the iQ SYBRgreen Supermix (Bio-Rad) on a Bio-Rad iCycler iQ instrument. About 10\% of each RT reaction was used to run real-time PCR reactions. The real-time PCR conditions were: $20^{\prime \prime}$ at $94^{\circ} \mathrm{C}, 30^{\prime \prime}$ at $59^{\circ} \mathrm{C}, 45^{\prime \prime}$ at $72^{\circ} \mathrm{C}$ for 45 cycles, followed by a melt curve cycle. Quantitative normalization of cDNA in each sample was performed using GAPDH as internal control. Samples were analysed in duplicate. Relative gene expression levels were determined by the comparative $C_{t}$ method. For RT real-time PCR experiments the following primer sets were used: HNRF-1 $\mathrm{F}$ sense 5'-CCAGACGACGCAAGCATCAG3', HNRF-1 R antisense 5'-GGGATCTGGACCAGGCCATT-3' (gene $n r f-1$ ); HTFAM F sense 5'-TGTTCACAATGGATAGGCAC-3' HTFAM $\mathrm{R}$ antisense $5^{\prime}$-TCTGGGTTTTCCAAAGCAAG-3' (gene $m t$ - $t f a$ ); RealESSS-F inEx2 sense 5'-ACCCAGACTCCCATGGTTATG-3', Real-163-R antisense $5^{\prime}$-GGACCACTCTTTCATCCCATCC-3' (gene ndufs 11); Glic-1 $\mathrm{F}$ sense 5'-GAAGGTGAAGGTCGGAGT-3', Glic-4 $\mathrm{R}$ antisense 5'-CATGGGTGGAATCATATTGGAA-3' (gene Gapdh); Cyt SOD F sense 5'-TGGTTTGCGTCGTAGTCTCCT-3', Cyt SOD R antisense 5'-AATGCTTCCCCACACCTTCA-3' (gene sod1); MnSOD F sense 5'-CTGGACAAACCTCAGCCCT-3', MnSOD $\mathrm{R}$ antisense 5'CTGATTTGGACAAGCAGCAA-3' (gene sod2); Catalase $\mathrm{F}$ sense 5'-TGGAAAGAAGACTCCCATCG-3', Catalase $\mathrm{R}$ antisense $5^{\prime}$ CCAGAAGTCCCAGACCATGT-3' (gene catalase); PGC-1alpha F sense 5'-AAACAGCAGCAGAGACAAATGC-3', PGC-1alpha $\mathrm{R}$ antisense 5'-TTGGTTTGGCTTGTAAGTGTTGTG-3' (gene pgc-1 alpha).

\subsection{Electron microscopy}

Monocytes, immature DC (iDC) and mature DC ( $\mathrm{mDC}$ ) were fixed in 3\% GTA (glutaraldehyde) in $0.1 \mathrm{M}$ sodium phosphate buffer ( $\mathrm{pH}$ 7.4) for $2 \mathrm{~h}$, washed in the same buffer and then postfixed in $1 \%$ $\mathrm{OsO}_{4}$ at $4{ }^{\circ} \mathrm{C}$. Afterward, cells were dehydrated in graded ethanol, and embedded in Epon $812.60 \mathrm{~nm}$ ultrathin sections were cut with a diamond knife on a LKB-V ultratome, stained with uranyl acetate followed by lead citrate and examined under a Zeiss EM 109 electron microscope (Zeiss, Oberkochen, Germany). Digital images were analysed and recorded with a cooled camera Gatan CMS (Gatan GmbH, München, Germany).

\subsection{Statistical analysis}

Results are expressed as mean \pm SEM. Statistical significance was determined by Student's $t$ test. Differences were considered significant when $p<0.05$.

\section{Results and discussion}

Ultrastructural analysis revealed a significant increment in the number of condensed mitochondria in immature DC compared to monocytes (Del Prete et al., 2008). To investigate whether the differentiation was accompanied by structural as well as functional changes in mitochondrial respiratory chain complexes, we analysed the expression level of protein subunits of respiratory complexes, as well as their specific enzymatic activities. Since loading of equal amount of protein in WB system did not show any major difference between monocytes and DC in the abundance of the mitochondrial complexes protein levels (Fig. 1A), proteins derived from an equal number of nuclei were loaded. This WB analysis method, currently used by others (Franko et al., 2008), can indeed take into account the protein synthesis occurring during DC differentiation and demonstrate the actual increase in mitochondrial proteins per nuclear gene copy. From this analysis, a statistically significant increase in the protein level of NDFUS3 of Complex I (1.79 fold), Core I of Complex III (2.28 fold), and Cox IV of Complex IV (2.07 fold) (Fig. 1B) was observed in DC compared to monocytes. The increase in the expression of protein subunits of respiratory enzymes was accompanied by changes of their catalytic function. In fact, as shown in Fig. 1C, the enzymatic activity of Complex I in monocytes, corresponding to $0.22 \pm 0.05 \mathrm{nmol} / \mathrm{min} / 10^{6}$ cells, increased to $1.36 \pm 0.20 \mathrm{nmol} / \mathrm{min} / 10^{6}$ cells in DC ( 6.18 fold). Similarly, DC showed 4.47 fold increase in the activity of Complex IV compared to monocytes $\left(6.74 \pm 0.46\right.$ vs $1.42 \pm 0.18 \mathrm{nmol} / \mathrm{min} / 10^{6}$ cells, respectively). In order to compare directly the expression of mitochondrial proteins between monocytes and DC, a new magnetic sorting method was used to isolate pure functional mitochondria from cultured cells, which turned out suitable also for a small number of human primary cells (Hornig-Do et al., 2009). WB analysis showed that the expression of the outer membrane proteins TOM22, VDAC, and NDUFS3 in mitochondria isolated from an equal nucleus number was significantly increased in DC compared to monocytes (Fig. 1D). Overall, these findings indicate that, during the differentiation process of monocytes into DC, mitochondrial activity is greatly enhanced, as revealed by the increase in the respiratory enzyme activities and in their protein expression levels.

In differentiated DC, mitochondrial oxidative phosphorylation system appears to provide most of cellular ATP production. In fact as shown in Fig. 2 the ATP content in differentiated DC increased progressively along with the differentiation process. Treatment with the respiratory chain inhibitor rotenone reduced markedly the ATP content, also causing a very large increase of lactate production. Conversely, in the absence of rotenone, lactate release in the culture medium was much lower. This strongly suggests that the increased electron transfer capacity upon differentiation implies a higher utilization of mitochondria for ATP synthesis. Interestingly, the activation process of $\mathrm{T}$ lymphocytes through CD28 signalling has been described to be associated mainly to enhanced glycolysis, with only a small proportion of glucose oxidized in mitochondria (Frauwirth et al., 2002). From these results it can be argued that cell differentiation and activation, which are definitely distinct processes, might exhibit different metabolic state.

We have previously shown that a peak of ROS production, lasting almost $1 \mathrm{~h}$, took place after the addition of growth factors to monocyte suspension. The presence of rotenone in the monocyte culture, as well as that of the classical antioxidant Catalase, caused a similar drop in ROS production and a suppression of the DC differentiation as revealed by a substantial reduction in CD1a expression. Under these conditions oxygen consumption activity measured at day +6 resulted to be strongly inhibited (Del Prete et al., 2008). These experiments provide evidence for a causality relationship between ROS and electron transfer activity accompanying mitochondrial biogenesis.

Furthermore, since the progressively increase of mitochondrial activity of DC, compared to monocytes, likely represents a higher potential of ROS production, it seems plausible that DC might be equipped with more efficient antioxidant enzymatic protection systems in order to detoxify ROS. To test this hypothesis, we analysed the activity and the expression of main anti-oxidant enzymes. Fig. 3 reports measurements of the activity of Catalase (A) and of the cytosolic (CuZn) and mitochondrial (Mn) superoxide 
A
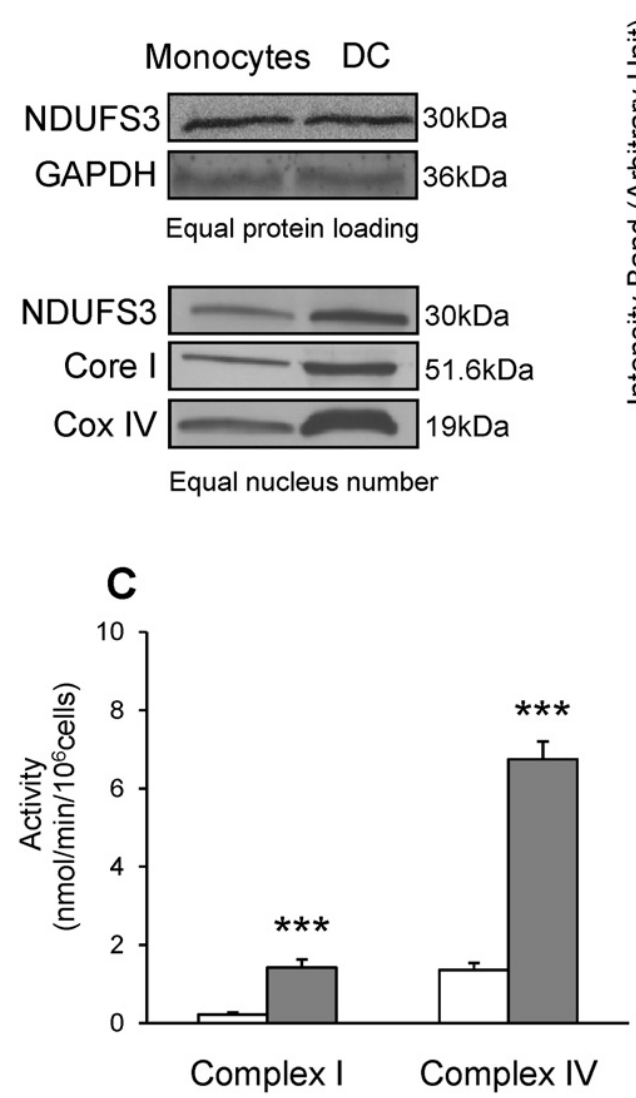

B

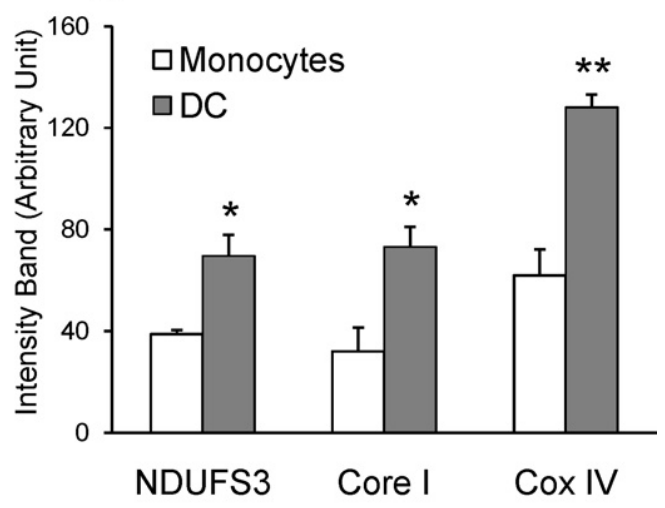

D

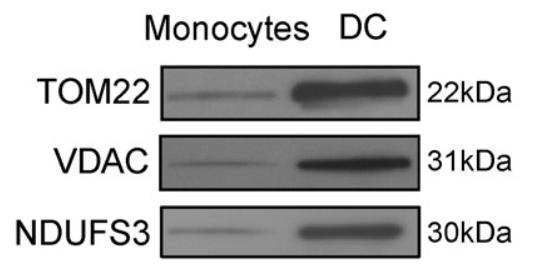

Fig. 1. Mitochondrial respiratory complexes during DC differentiation. (A) WB analysis of mitochondrial respiratory complex proteins in monocytes and DC. Equal amount of proteins $\left(50 \mu \mathrm{g}\right.$, upper part) or proteins corresponding to equal number of cells $\left(2 \times 10^{6}\right.$, lower part) from a representative donor sample were loaded and the membranes were probed for the indicated proteins. (B) Quantitative data derived from the densitometric analysis of the bands is reported in (A). Values are reported as mean \pm SEM $(n=4)$. (C) Enzymatic activities of Complex I and Complex IV as nmol/min $/ 10^{6}$ monocytes and DC. Values are reported as mean \pm SEM $(n=4)$. (D) WB analysis of TOM22, VDAC and NDUFS3 from mitochondrial fraction isolated from $6 \times 10^{6}$ monocytes and DC (DC/monocytes: TOM22, 6.6; VDAC, 8.7; NDUFS3, 6.9 fold). One experiment out of three is represented $\left({ }^{*} p<0.05,{ }^{* *} p<0.01,{ }^{* * *} p<0.001\right.$, DC vs monocytes).
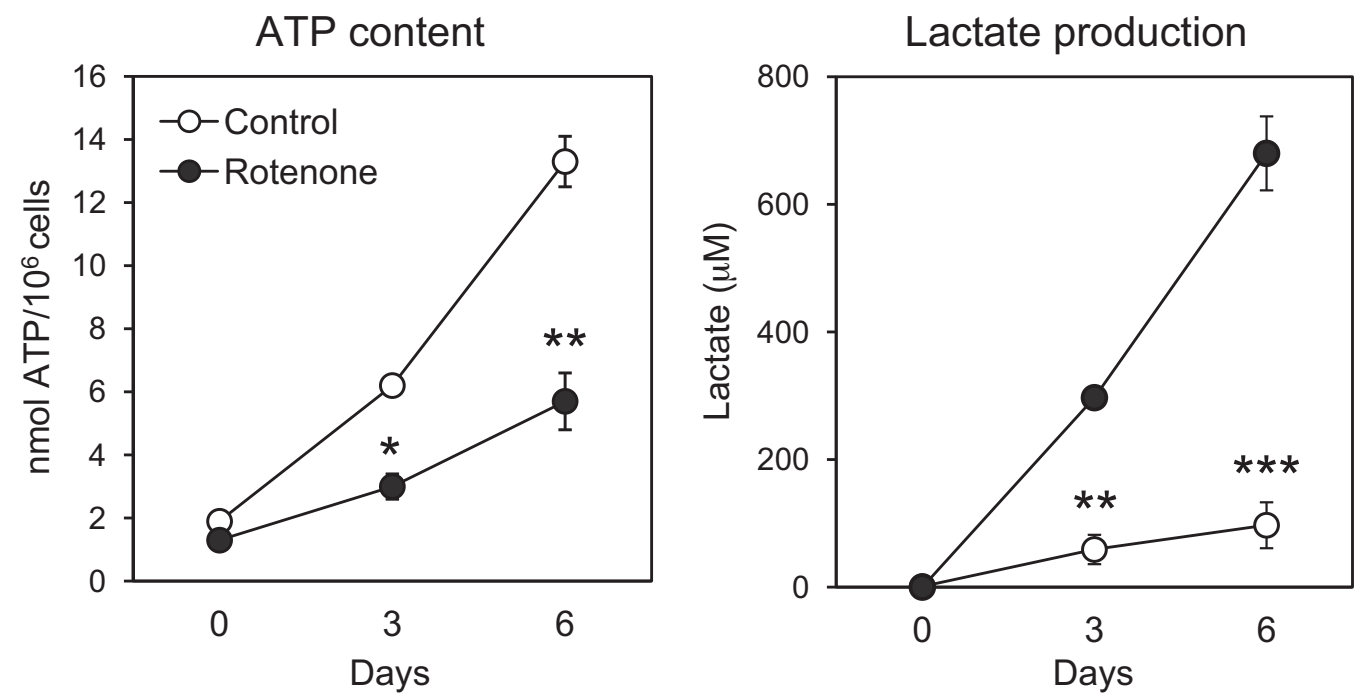

Fig. 2. ATP content and lactate production evaluated in the absence and in the presence of the respiratory chain inhibitor rotenone. ATP levels expressed as nmol ATP $/ 10^{6}$ cells and lactate released in the culture medium $(\mu \mathrm{M})$ were measured in monocytes (day 0$)$, at intermediate time point (day 3 ) and in DC (day 6 ), in the absence and in the presence of rotenone. The mean \pm SEM of at least 3 independent experiments are indicated $\left({ }^{*} p<0.05,{ }^{* *} p<0.01,{ }^{* * *} p<0.001\right.$, DC rotenone vs DC control). 
A

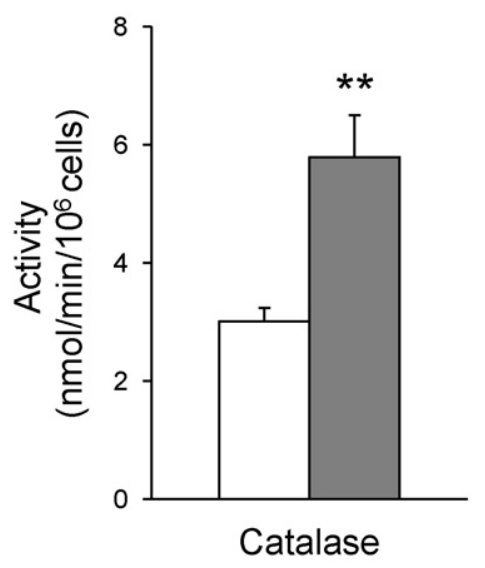

C

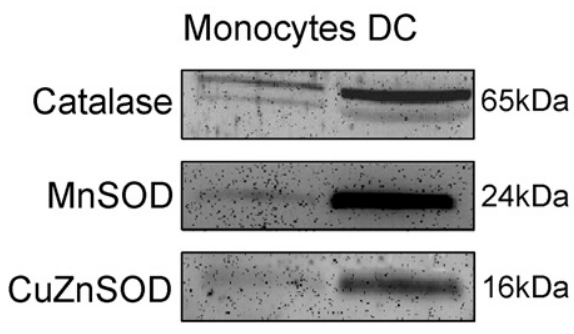

B
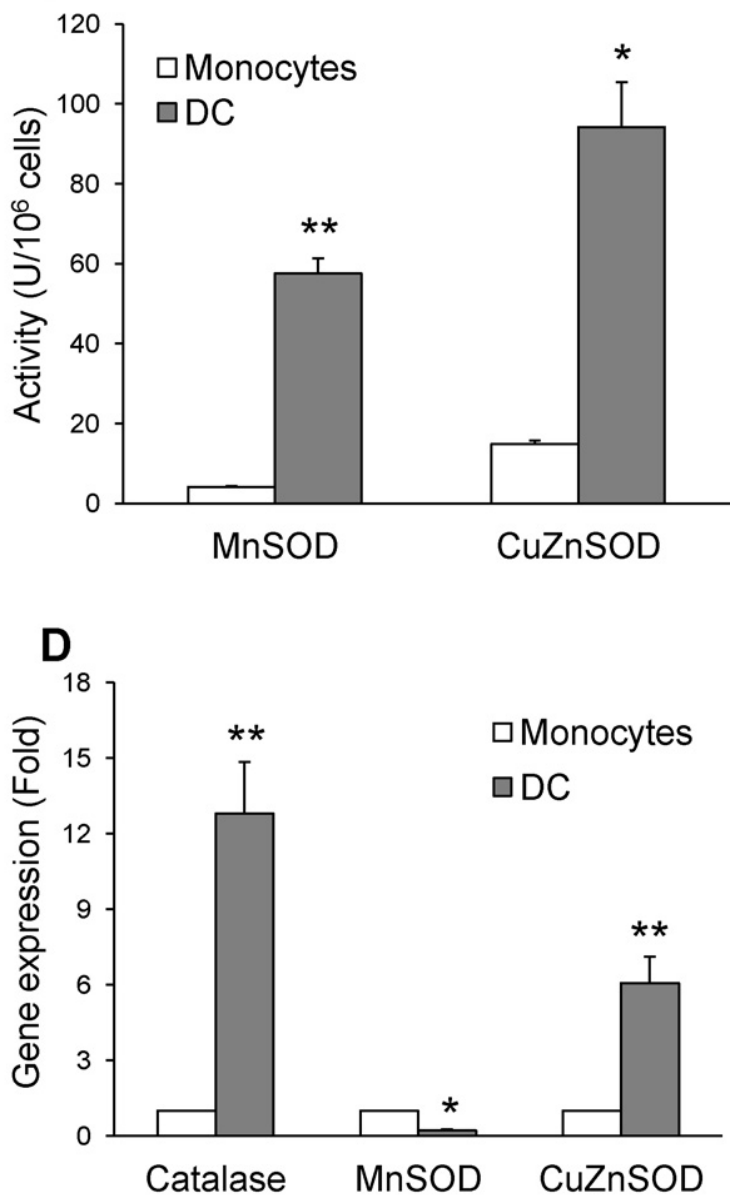

Fig. 3. Anti-oxidant enzyme activity and expression during DC differentiation. Activity of Catalase (A) and MnSOD and CuZnSOD (B) in monocytes and DC. Results are indicated as mean $\pm \operatorname{SEM}(n=4)$. (C) WB analysis of proteins from an equal number of monocytes and DC (one experiment out of three is represented). (D) Gene expression of Catalase, MnSOD and CuZnSOD by real-time qPCR experiments. Results are expressed as fold increase (DC/monocytes). Values are reported as mean \pm SEM $(n=4)\left({ }^{*} p<0.05\right.$, ${ }^{* *} p<0.01$, DC vs monocytes).

dismutase (B). The enzymatic activity of Catalase in monocyte precursors (approximately $3 \mathrm{nmol} / \mathrm{min} / 10^{6}$ cells) increased about two fold upon differentiation. The analysis of the enzymatic activity of SOD revealed that the mitochondrial MnSOD form increased much more $\left(4.11 \pm 0.39\right.$ vs $57.78 \pm 5.3 \mathrm{U} / 10^{6}$ cells, monocytes vs DC) than the cytosolic form ( $14.88 \pm 0.88$ vs $94.2 \pm 15.9 \mathrm{U} / 10^{6}$ cells, monocytes vs DC), suggesting that a more intense anti-oxidant activity occurs in the mitochondrial compartment of DC. WB analysis confirmed the above data. In fact, densitometric analysis showed a relevant increase ( $30.4 \pm 6.9$ fold) in MnSOD protein expression, while the cytosolic enzymes, Catalase and CuZnSOD, resulted only slightly increased upon DC differentiation $(2.8 \pm 0.7$ fold and $4.7 \pm 0.1$ fold, respectively) (Fig. 3C).

Changes in anti-oxidant enzyme gene expression occurring during DC differentiation is shown in Fig. 3D. While the relative gene expression of the cytosolic enzymes Catalase and CuZnSOD revealed a significant increase in DC compared to monocytes (12.7 and 6 fold, respectively), no induction was observed in the RNA level of MnSOD ( 0.21 fold). This apparently contrasting observation that a major induction of MnSOD is seen at the protein level than at the RNA level has been also reported previously (Angenieux et al., 2001 ) and explained considering that the localization into the mitochondrial matrix would protect the mature mitochondrial protein from proteolytic degradation. Consequently, it can be deduced that during DC differentiation process, taking place in 6-days culture, large increase in the concentration of mitochondrial proteins, such as MnSOD, can occur from marginal increase of the corresponding mRNA levels (Angenieux et al., 2001). Overall, these findings confirmed previous observations (Rivollier et al., 2006) showing that DC are equipped with a very efficient anti-oxidant systems that may assure higher resistance to oxidative environments.

The higher efficiency of antioxidant apparatus we found in mitochondria as compared to the cytosolic compartment of DC may reflect a condition where most of superoxide is produced in the matrix. It has in fact been reported that Complex I generated superoxide is released exclusively into the matrix, together with $50 \%$ of the superoxide produced by Complex III (Muller et al., 2004). On the other hand, the key role of the mitochondrial SOD is illustrated by the lethal phenotype of mice lacking the matrix superoxide dismutase (Sod2) gene (Li et al., 1995). Finally, as described for osteogenic differentiation of human mesenchymal stem cells (Chen et al., 2008), our observations suggest that during DC differentiation a coordinated regulation between mitochondrial biogenesis and anti-oxidant defence systems needs to be orchestrated in order to let ROS production trigger differentiation but, at the same time, prevent accumulation of ROS when aerobic mitochondrial metabolism becomes dominant.

To clarify the mechanisms underlying the regulation of mitochondrial biogenesis occurring during DC differentiation, we investigate the mtDNA copy number and the mRNA expression 

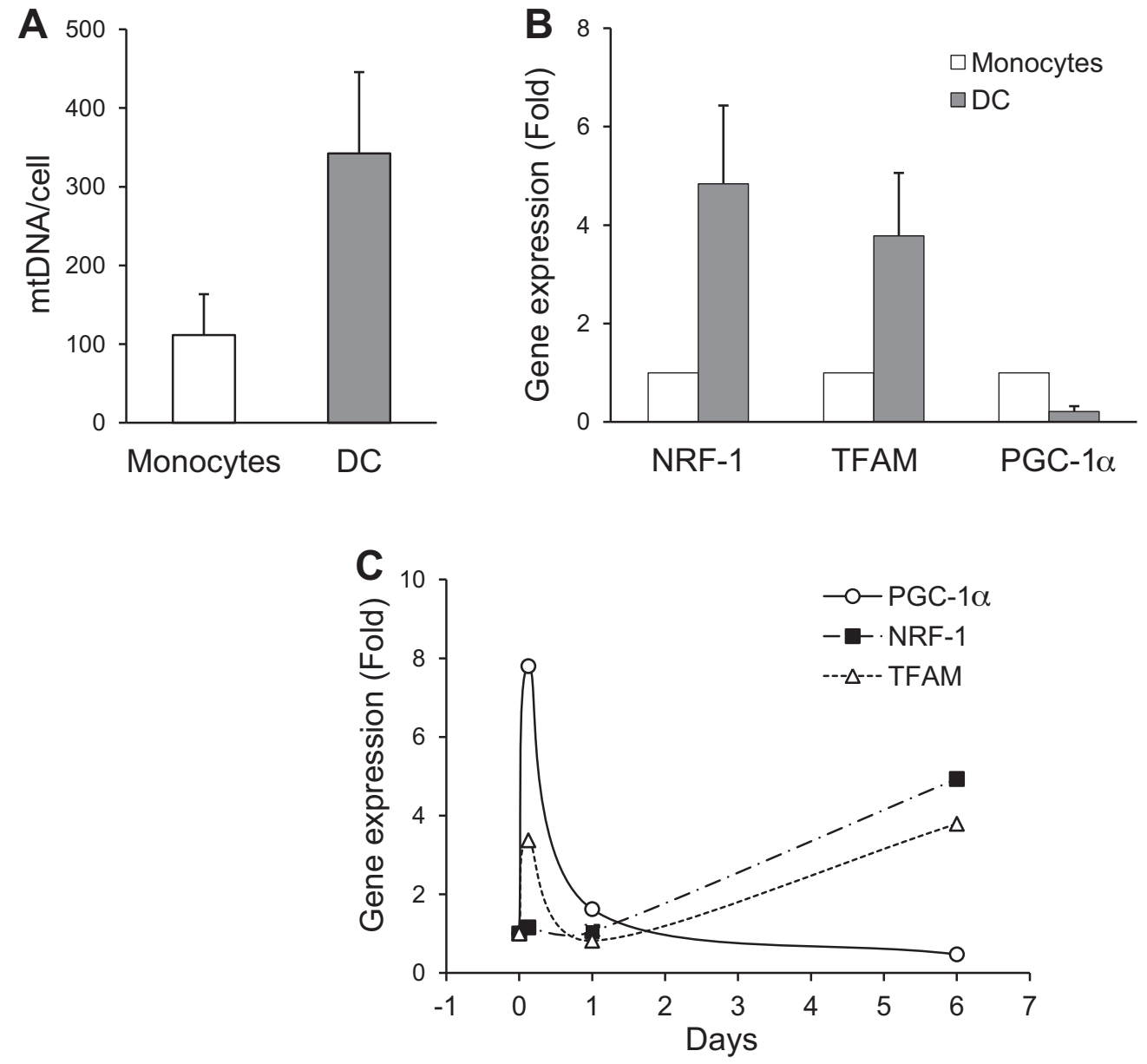

Fig. 4. Mitochondrial biogenesis occurring during DC differentiation. (A) mtDNA copy number per cell. Values are reported as mean $\pm S E M(n=4)$. (B) $m R N A$ levels of NRF-1, TFAM, and PGC- $1 \alpha$. mRNA levels were determined by RTqPCR of total RNA normalized to GAPDH. Results are expressed as fold increase (DC/monocytes). Data are means \pm SEM of independent determinations $(n=4)$. (C) Kinetic study (day $0,+1$ and +6 ) of mRNA levels of the three genes. Results from a representative experiment are expressed as fold increase.

levels of the main mitochondrial biogenesis-associated genes, including PGC-1 $\alpha$, TFAM, and NRF-1, both in monocyte precursors and in DC. Real-time qPCR experiments revealed that mtDNA copies were approximately increased by 3.5 fold in DC compared to monocytes (Fig. 4A). The increase of mtDNA was associated with the triggering of the mitochondrial biogenesis activation pathway. In fact, the gene expression of NRF-1 and TFAM (Fig. 4B) was significant increased in DC compared to monocytes (NRF-1, 4.84 and TFAM, 3.78 fold). Unexpectedly, at the end of the differentiation process, DC showed very low level of PGC- $1 \alpha$ gene expression, even lower than monocyte precursors ( 0.21 fold). However, a kinetic study of the three main mitochondrial biogenesis-associated genes revealed that the peak in PGC- $1 \alpha$ gene expression was suddenly reached $3 \mathrm{~h}$ after the addition of specific DC differentiation soluble factors (GM-CSF and IL-4) in monocyte culture medium. PGC- $1 \alpha$ expression rapidly declined after $24 \mathrm{~h}$ culture, while NRF- 1 and TFAM showed a gradual increase in their expression culminating at the end of the differentiation process (Fig. 4C). These findings suggest that an early input by the master regulator of mitochondrial biogenesis PGC- $1 \alpha$ is needed to trigger the subsequent activation of the downstream transcription factors, NRF-1 and TFAM, during DC differentiation.

Ultrastructural features of mature $\mathrm{DC}(\mathrm{mDC})$ compared to immature DC (iDC) and monocytes, together with the expression levels of both mitochondrial biogenesis proteins and respiratory enzymes were analysed. No substantial differences were observed between iDC and $\mathrm{mDC}$ on the ultrathin sections (Fig. 5B and C). DC appeared as elongated or flattened cells with thin plasmamembranes filopodia and eucromatic nuclei, containing numerous rounded or rod-like mitochondria, in a typical condensed form (Fig. 5E and F). Monocytes showed both condensed and orthodox mitochondria, these latter having a thin inter-membrane space and lamellar cristae (Fig. 5A and D). The quantitative analysis of mitochondria of the different cell types is reported in Fig. 5G. From morphometric analysis no significant differences in the condensed mitochondria number were observed between iDC and $\mathrm{MDC}$, which were, on the other hand, significantly increased compared to monocytes. Thus the final DC maturation step does not seem to be accompanied by more active mitochondrial biogenesis.

In conclusion, what is emerging from these and the previously reported data (Del Prete et al., 2008) is that the sudden ROS production upon addition of the differentiation factors to the monocyte precursor suspension induces a rapid increase of PGC- $1 \alpha$, a process lasting less than one out the six days required for their differentiation into DC. Since the increase of mitochondrial number/activity induced by PGC- $1 \alpha$ may cause in principle an increase of ROS production, then the protein plays a role in the regulation of the ROS metabolic program, leading to over-expression of ROS detoxifying enzymes, in particular MnSOD (St-Pierre et al., 2006). ROS level in the matrix, where superoxide is mostly released, is thus maintained definitely low to have cells functioning normally. In summary, this study demonstrates, for the first time, that an active 

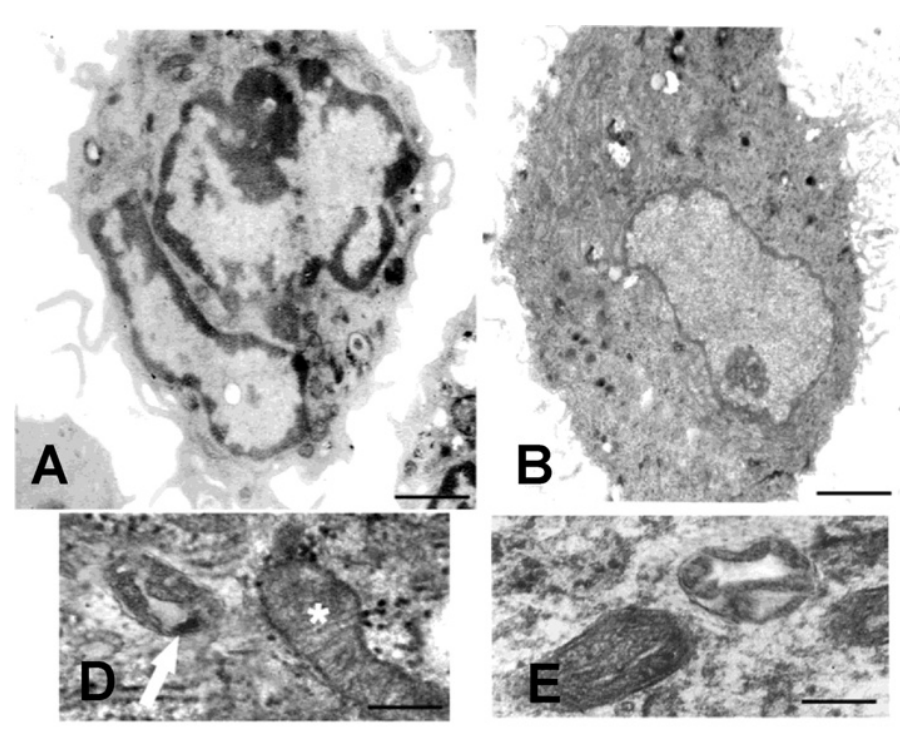

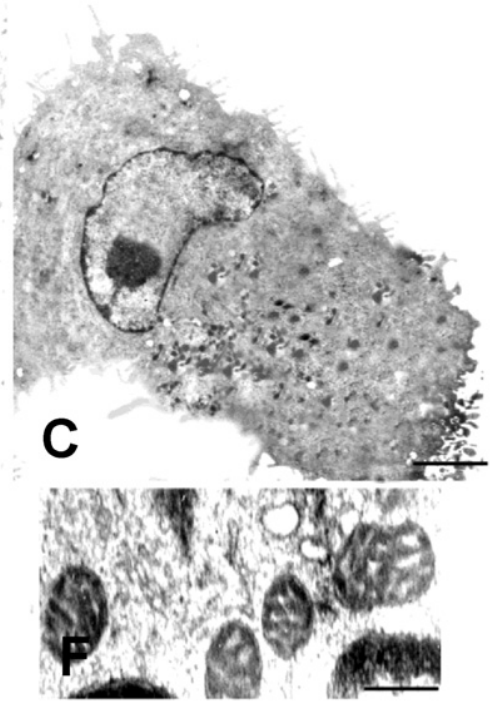

G

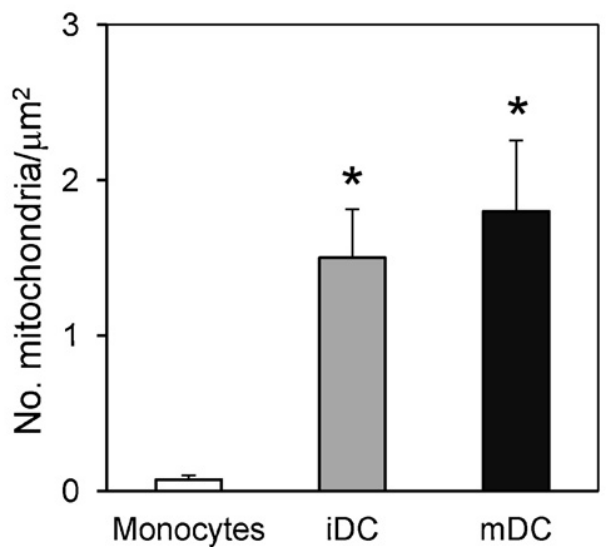

H

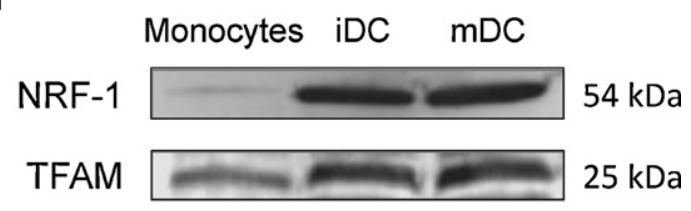

NDUFS3 $=-2$

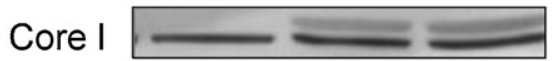

Cox IV

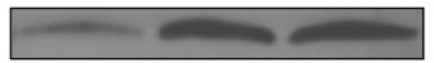

Fig. 5. TEM analysis, respiratory chain enzyme expression and mitochondrial biogenesis proteins in mDC, iDC and monocytes. Ultrastructural analysis of monocytes (A), immature (B) mature DC (C). DC (B, C) lined by the plasmamembrane with fillipodies and pseudopodes show the cytoplasm with numerous condensed mitochondria (E, F). Monocytes show mitochondria with orthodoxic (asterisk) and condensed (arrow) conformation (A, D). Scale bars (original magnification): A-C, $1.42 \mu \mathrm{m}$; D-F, $0.25 \mu \mathrm{m}$. (G) For the morphometric analysis, mitochondria of 10 cells for each sample were counted on the electron micrographs at final magnification of $12,000 \times$ by computer-aided analysis. The mean value for all the micrographs \pm SEM are indicated $\left({ }^{*} p<0.05\right.$, iDC and mDC vs monocytes). (H)WB analysis of mitochondria biogenesis and respiratory complex protein subunits in monocytes, iDC and $\mathrm{mDC}$ from a representative donor sample out of three experiments.

mitochondrial biogenesis occurs during the differentiation process of monocytes into DC. Moreover, the fine regulation between PGC$1 \alpha$ and the expression of mitochondrial ROS-detoxifying enzymes might represent a potential target in those pathological conditions where DC functions and metabolic alterations are simultaneously involved.

\section{Disclosures}

No conflicts of interest are declared by the authors.

\section{Acknowledgements}

This work was financially supported by National Research Projects (PRIN) from MIUR (Ministero dell'Istruzione Università e Ricerca), and Italian Ministry of Health (Progetto Giovani Ricercatori 2007). We thank Dr. Susanna D’Oria and Dr. Anna Abbrescia for technical assistance and Dr. Elisabetta Crollo from the local blood bank.

\section{References}

Angenieux C, Fricker D, Strub JM, Luche S, Bausinger H, Cazenave JP, et al. Gene induction during differentiation of human monocytes into dendritic cells: an integrated study at the RNA and protein levels. Functional and Integrative Genomics 2001;1:323-9.

Banchereau J, Briere F, Caux C, Davoust J, Lebecque S, Liu YJ, et al. Immunobiology of dendritic cells. Annual Review of Immunology 2000;18:767-811.

Chan DC. Mitochondria: dynamic organelles in disease, aging, and development. Cel 2006;125:1241-52.

Chen CT, Shih YR, Kuo TK, Lee OK, Wei YH. Coordinated changes of mitochondrial biogenesis and antioxidant enzymes during osteogenic differentiation of human mesenchymal stem cells. Stem Cells 2008;26:960-8.

Clayton DA. Nuclear-mitochondrial intergenomic communication. Biofactors 1998;7:203-5.

Del Prete A, Zaccagnino P, Di Paola M, Saltarella M, Oliveros Celis C, Nico B, et al. Role of mitochondria and reactive oxygen species in dendritic cell differentiation and functions. Free Radical Biology and Medicine 2008;44:1443-51.

Franko A, Mayer S, Thiel G, Mercy L, Arnould T, Horning-Do HT, et al. CREB-1alpha is recruited to and mediates upregulation of the cytochrome $\mathrm{c}$ promoter during enhanced mitochondrial biogenesis accompanying skeletal muscle differentiation. Molecular and Cellular Biology 2008;28:2446-59.

Frauwirth KA, Riley JL, Harris MH, Parry RV, Rathmell JC, Plas DR, et al. The CD28 signaling pathway regulates glucose metabolism. Immunity 2002;16:769-77.

Garesse R, Vallejo CG. Animal mitochondrial biogenesis and function: a regulatory cross-talk between two genomes. Gene 2001;263:1-16. 
Goffart S, Wiesner RJ. Regulation and co-ordination of nuclear gene expression during mitochondrial biogenesis. Experimental Physiology 2003;88:33-40.

Hornig-Do HT, Gunther G, Bust M, Lehnartz P, Bosio A, Wiesner RJ. Isolation of functional pure mitochondria by superparamagnetic microbeads. Analytical Biochemistry 2009;389:1-5.

Kelly DP, Scarpulla RC. Transcriptional regulatory circuits controlling mitochondrial biogenesis and function. Genes and Development 2004;18:357-68.

Kiefel BR, Gilson PR, Beech PL. Cell biology of mitochondrial dynamics. International Review of Cytology 2006;254:151-213.

Kroemer G, Reed JC. Mitochondrial control of cell death. Nature Medicine 2000;6:513-9.

Li Y, Huang TT, Carlson EJ, Melov S, Ursell PC, Olson JL, et al. Dilated cardiomyopathy and neonatal lethality in mutant mice lacking manganese superoxide dismutase. Nature Genetics 1995;11:376-81.

Muller FL, Liu Y, Van Remmen H. Complex III releases superoxide to both sides of the inner mitochondrial membrane. Journal of Biological Chemistry 2004;279:49064-73.

Pacelli C, De Rasmo D, Signorile A, Grattagliano I, di Tullio G, et al. Mitochondrial defect and PGC-1alpha dysfunction in parkin-associated familial Parkinson's disease. Biochimica et Biophysica Acta 2011;1812:1041-53.

Rivollier A, Perrin-Cocon L, Luche S, Diemer H, Strub JM, Hanau D, et al. High expression of antioxidant proteins in dendritic cells: possible implications in atherosclerosis. Molecular and Cellular Proteomics 2006;5: 726-36.
Sattler M, Winkler T, Verma S, Byrne CH, Shrikhande G, Salgia R, et al. Hematopoietic growth factors signal through the formation of reactive oxygen species. Blood 1999;93:2928-35.

Scarpulla RC. Metabolic control of mitochondrial biogenesis through the PGC-1 family regulatory network. Biochimica et Biophysica Acta 2011;1813:1269-78.

Scarpulla RC. Nuclear activators and coactivators in mammalian mitochondrial biogenesis. Biochimica et Biophysica Acta 2002;1576:1-14.

Scarpulla RC. Nuclear control of respiratory chain expression by nuclear respiratory factors and PGC-1-related coactivator. Annals of the New York Academy of Sciences 2008; 1147:321-34.

Sgobbo P, Pacelli C, Grattagliano I, Villani G, Cocco T. Carvedilol inhibits mitochondrial complex I and induces resistance to $\mathrm{H}_{2} \mathrm{O}_{2}$-mediated oxidative insult in H9C2 myocardial cells. Biochimica et Biophysica Acta 2007;1767:222-32.

Sheng KC, Pietersz GA, Tang CK, Ramsland PA, Apostolopoulos V. Reactive oxygen species level defines two functionally distinctive stages of inflammatory dendritic cell development from mouse bone marrow. Journal of Immunology 2010;184:2863-72.

Sozzani S. Dendritic cell trafficking: more than just chemokines. Cytokine and Growth Factor Reviews 2005;16:581-92.

St-Pierre J, Drori S, Uldry M, Silvaggi JM, Rhee J, Jager S, et al. Suppression of reactive oxygen species and neurodegeneration by the PGC-1 transcriptional coactivators. Cell 2006; $127: 397-408$.

Steinman RM. Some interfaces of dendritic cell biology. APMIS 2003;111(7-8):675-97. 